\title{
A COUNTEREXAMPLE TO THE PARAMAGNETIC CONJECTURE
}

\author{
J. AVRON and B. SIMON ${ }^{1}$ \\ Department of Physics, Technion - Israel Institute of Technology, Haifa, Israel
}

Received 18 August 1979

We provide a counterexample to the universal paramagnetism conjecture of Hogreve, Schrader and Seiler. The counterexample is based on the Bohm-Aharonov effect.

Several years ago, one of us [1] proved an inequal. ity expressing the universal diamagnetic tendency of spinless bosons. For a single particle in external (local) electric potential $V$ and magnetic potential $a$, the inequality can be expressed as follows: Let

$H_{1}(a, V) \equiv(-\mathrm{i} \nabla-a)^{2}+V$

and let

$E_{1}(a, V) \equiv \inf \operatorname{spec}\left[H_{1}(a, V)\right]$.

Then [1]:

$E_{1}(a, V) \geqslant E_{1}(a=0, V)$,

for any $a, V$. Subsequently, motivated by remarks of Nelson, Simon [2] extended (3) to a finite temperature result:

$\operatorname{Tr}\left(\exp \left(-\beta H_{1}(a, V)\right)\right) \leqslant \operatorname{Tr}\left(\exp \left(-\beta H_{1}(a=0, V)\right)\right)$

(see ref. [3] for further developments). Of course (4) implies (3) by taking $\beta \rightarrow \infty$.

Foughly one year ago, Hogreve et al. [4] put forward a very attractive conjecture about the situation when spin is taken into account. Let $\sigma$ be the conventional Pauli matrices

$$
\begin{aligned}
& H_{2}(a, V) \equiv(-\mathrm{i} \not \vec{D}-\not d)^{2}+V \\
& \quad=(-\mathrm{i} \nabla-a)^{2}+V+\sigma \cdot B,
\end{aligned}
$$

where $A=\Sigma_{i} A_{i} \sigma_{i}$ as usual. Then ref. [4] conjectures

1 Permanent address: Depts. of Mathematics and Physics, Princeton University, Princeton, NJ; research partially supported by USNSF Grant MCS-78-01885. (eq. (18) of ref. [4]) that

$\operatorname{Tr}\left(\exp \left(-\beta H_{2}(a, V)\right)\right) \geqslant \operatorname{Tr}\left(\exp \left(-\beta H_{2}(a=0, V)\right)\right)$

and, in particular, that

$E_{2}(a, V) \leqslant E_{2}(a=0, V)$.

In ref. [4] a number of arguments are given in favor of this conjecture and since the apperance of ref. [4] a number of interesting developments have tended to support the conjecture. First, an inequality on functional determinants which follows from (6) (and which was the main concern of ref. [4]) has been proven even for suitable Yang-Mills fields [5]. Moreover, for the special case $\boldsymbol{a}=\frac{1}{2}\left(\boldsymbol{B}_{0} \times \boldsymbol{r}\right)$ (i.e. $\boldsymbol{B}=\mathrm{a}$ constant, $B_{0}$ ), where (7) had been independently conjectured (in an equivalent form) by Avron et al. [6], Lieb ${ }^{\ddagger 1}$ proved that (7) held (it is still unknown whether (6) holds in this case). Subsequently, Avron and Seiler [7] extended Lieb's result to certain polynomial $B$ 's.

Our goal here is to provide a counterexample to (7) and thus to (6). We will deal with two dimensions and allow $V$ to be infinite in certain regions but given that (7) is false in that case it is easy to conclude (7) will be false for suitable three-dimensional cases with $V$ everywhere finite. Indeed, in the three-dimensional case, consider a potential $w_{a, l}(x, y, z)=V_{a}(x, y) \chi_{l}(z)$, where $V_{a}(x, y)$ $=\min (\dot{a}, V(x, y))$ and $\chi$ is the function which is 1 (respectively 0 ) for $|z|<l$ (respectively $|z| \geqslant l$ ). Then as $a, l \rightarrow \infty$, the ground state energy of the three-dimensional system approaches that of the two-dimensional system so if (7) holds for all three-dimensional systems

\$1 Lieb's proof appears as an appendix of ref. [6]. 
with finite potentials, it will hold for two-dimensional systems with potentials allowed to be infinite.

We will take a magnetic field $B(x, y) \neq 2$ which is axially symmetric under rotations in the plane centered at $(x, y)=(0,0)$. In this case a convenient gauge for $a$ is

$\boldsymbol{a}(\boldsymbol{\rho})=\left(2 \pi \rho^{2}\right)^{-1} \boldsymbol{\Phi}(\rho) \times \boldsymbol{\rho}$,

where $\Phi(\rho)$ is the total flux through the circle of radius $\rho^{\ddagger 3}$. The gauge (8) has $\operatorname{div} \boldsymbol{a}=0$. Thus ${ }^{\ddagger 4}$ :

$$
\begin{aligned}
& H_{2}(a, V)=(-\mathrm{i} \nabla-a)^{2}+V+\boldsymbol{\sigma} \cdot B \\
& \quad=\boldsymbol{p}^{2}+a^{2}+2 a \cdot \boldsymbol{p}+V+\boldsymbol{\sigma} \cdot B \\
& \quad=\boldsymbol{p}^{2}+\boldsymbol{a}^{2}-\left(2 \pi \rho^{2}\right)^{-1} \Phi(\rho) L_{z}+V+\sigma_{z} B .
\end{aligned}
$$

Now take $B=\lambda \widetilde{B}$ where $\lambda$ is a coupling constant which we will vary and $\widetilde{B}$ is the field which is 1 in a disc of radius 1 and 0 outside the disc. Now let $V=\rho^{2}+\mu W$ where $W$ is 1 (respectively 0 ) inside (respectively outside) the disc and take $\mu \rightarrow \infty$. If (7) holds for all finite $\mu$ it will hold in the limit. In this limit:

$H_{2}(\lambda)=p_{\mathrm{D}}^{2}+\lambda^{2} \widetilde{a}^{2}-\lambda\left(2 \pi \rho^{2}\right)^{-1} \widetilde{\Phi}(\rho) L_{z}+\rho^{2}$,

where $p_{\mathrm{D}}^{2}$ indicates the vanishing boundary condition on the circle of radius 1 . The $B$ term has dropped out since $B \neq 0$ only in the region where $V=\infty$. When $\lambda$ $=0, H_{2}(\lambda)$ has a ground state with $L_{z}=0$. Since $H_{2}(\lambda)$ is rotationally invariant, and the ground state has a finite distance from all other states, the ground state of $H_{2}(\lambda)$ will have $L_{z}=0$ for small $\lambda$. But then, since $\tilde{a}^{2}$ is strictly positive,

$E_{2}(\lambda)>E_{2}(\lambda=0)$

for $\lambda$ small, violating ( 7 ).

Clearly our counterexample is based on the old idea of Bohm-Aharonov [8]. We came upon it since in trying to verify (6) by writing the trace as a Wiener integral there are two terms which enter in the action

$\neq 2$ The field in two dimensions is a scalar but it is convenient to think of it as pointing in a fictitious third dimension and using three-vector notation.

$\neq 3 \Phi$ is a vector in the fictitious third dimension.

$\neq 4$ We abuse notation and use $\Phi(\rho)$ in eq. (9) as the magnitude of $\Phi$. when $B$ is turned on: $\int \boldsymbol{\sigma} \cdot \boldsymbol{B}(\boldsymbol{\omega}(t)) \mathrm{d} t$, which tends to increase the trace and i $\int a(\omega(t)) d \omega$ (Ito stochastic integral) which tends to decrease it. Since only closed paths enter the trace one is tempted to write $\int a(\omega)$ $X \mathrm{~d} \omega=$ flux within $\omega^{\neq 5}$ and it is clear that one would have to cancel effects of the field within $\omega$ by the field on $\omega$ which leads naturally to Bohm-Aharonov considerations. It is also clear from this point of view that for the $V$ we discuss and $\beta$ finite that (6) fails ${ }^{\ddagger 6}$.

Finally we remark that Lieb's proof ${ }^{\ddagger 1}$ in the case $B=B_{0}$ depends on the infinite degeneracy of the ground state of $H_{1}(a, V=0)$. For the $B$ we pick there is no normalizable ground state for $\lambda$ small (see ref. [9]).

One of us (B.S.) would like to thank the Technion Physics Department for its hospitality and also the Egged bus company.

*5 Since $\omega$ is a general brownian path which is not rectifiable (see, e.g., Simon [9]), one cannot really talk about the flux through $\omega$ for general $\omega$ but it is a useful intuition.

$\neq 6$ Since $V=\infty$ in the region $B \neq 0$, the flux is just a winding number which can be defined for any continuous $\omega$ which avoids the region where $V=\infty$. If $A_{n} \geqslant 0$ is the contribution to the trace when $a=0$ at paths with winding number $n$, then the right side of eq. (6) $=A_{n}$ and the left side of eq. (8) $=\Sigma \mathrm{e}^{\mathrm{in} \Phi} A_{n}$ and eq. (6) is obviously false.

\section{References}

[1] B. Simon, Phys. Rev. Lett. 36 (1976) 804.

[2] B. Simon, Ind. Math. J. 26 (1977) 1067.

[3] H. Hess, R. Schrader and D. Uhlenbrock, Duke Math. J. 44 (1977) 893;

B. Simon, J. Op. Th. 1 (1979), to be published; B. Simon, J. Func. Anal., to be published.

[4] H. Hogreve, R. Schrader and R. Seiler, Nucl. Phys. B142 (1978) 525.

[5] D. Brydges, J. Frohlich and E. Seiler, Ann. Phys., to be published.

[6] J. Avron, I. Herbst and B. Simon, Duke Math. J. 45 (1978) 847.

[7] J. Avron and R. Seiler, Phys. Rev. Lett. 42 (1979) 931.

[8] Y. Aharonov and D. Bohm, Phys. Rev. 115 (1959) 485.

[9] B. Simon, Functional integration and quantum physics (Academic Press, 1979).

[10] A. Casher and Y. Aharonov, Phys. Rev. A19 (1979) 2461. 